Original Research Article

\title{
Comparative evaluation of bivalent malaria rapid diagnostic tests versus traditional microscopy method in assessment of malaria in blood donors at a tertiary care teaching hospital and regional blood transfusion centre in central India
}

\author{
Jain R', Jain P², Kashiv $\mathbf{M}^{3}$, P. Desai ${ }^{4}$, U. Chudgar ${ }^{5}$, N. Choudhury ${ }^{6}$, V.K. Mahadik ${ }^{7}$ \\ ${ }^{1}$ Dr. Roopam Jain, ${ }^{2}$ Dr. Preeti Jain, ${ }^{3}$ Dr. Mohit Kashiv, above authors are attached with C R Gardi Hospital and R. D, \\ Gardi Medical College, Ujjain, ${ }^{4}$ Dr. P. Desai, Tata Memorial Hospital, Parel, Mumbai, ${ }^{5}$ Dr. U. Chudgar, Prathama Blood \\ Centre, Ahmedabad, ${ }^{6}$ Dr. N. Choudhury, Fortis Memorial Hospital, Delhi, ${ }^{7}$ Dr. V.K. Mahadik, C.R.Gardi Hospital and R \\ D Gardi Medical College, Ujjain, MP, India.
}

Corresponding Author: Dr. Preeti Jain, C R Gardi Hospital and R. D, Gardi Medical College, Ujjain, MP, India. Email: drroopamjain@gmail.com

\begin{abstract}
Background: Global malaria control efforts are based on 2 broad components: vector control and improved diagnosis and treatment of patients with clinical malaria. Until recently, conventional diagnosis of malaria has been based on either clinical diagnosis or use of microscopic examination of peripheral blood smears. The microscopic detection of blood though considered the gold standard for malaria diagnosis, it is quite laborious and require adequate technical skill and man power. This had urged the development of other microscopic malarial and rapid detection test based on the detection of malarial parasite antigen in the blood. The purpose of this study is to evaluate results of Rapid diagnostic tests for malaria and to corroborate the results with microscopy. Material and Method: This study targeted 10,310 units of donor blood, which were screened for malaria by RDT during the period of February, 2017 to April, 2018 at Tertiary Care Teaching Hospital and Blood Transfusion Centre, R D Gardi Medical College, Ujjain. Peripheral smears were analyzed to confirm the malaria parasite. Result: Nineteen $(0.18 \%)$ donors were found to be malaria antigen positive, of which only three $(15.8 \%)$ were confirmed by microscopy. None of the donor had given a history of fever/malaria during pre-donation screening. Seasonal variations were observed. Conclusion: Although RDT is an important tool for malaria testing in rural settings, we suggest the diagnosis must be confirmed with microscopy method. RDTs can be an important tool for malaria testing, peripheral smear microscopy continues to be the gold standard diagnostic test for malaria diagnosis.RDT can be an important tool for malaria testing, peripheral smear microscopy continues to be the gold standard diagnostic test for malaria diagnosis.
\end{abstract}

Keywords: Blood Donors, RDTs, Peripheral microscopy

\section{Introduction}

In India, it is mandatory to test every unit of blood collected for hepatitis B, hepatitis C, HIV, syphilis and malaria [1]. If donors test positive to any of the five infections, their blood is discarded. Transmission of malaria by blood transfusion was one of the first recorded incidents of transfusion transmitted infection [2].

The frequency of transfusion-transmitted malaria varies from 0.2 cases per million in nonendemic countries to 50 or more cases per million in endemic areas [3].

Manuscript received: $26^{\text {th }}$ July 2018

Reviewed: $4^{\text {th }}$ August 2018

Author Corrected: $10^{\text {th }}$ August 2018

Accepted for Publication: $16^{\text {th }}$ August 2018
Importance of recognizing transfusion-transmitted malaria lies in the fact that it can lead to febrile transfusion reaction which can falsely simulate a hemolytic transfusion reaction. It can lead to the wide spread dissemination and spread of drug-resistant malarial parasite [4]

Blood transfusion possesses a problem because the parasites keep their infective activity for at least 14 days in blood bottles stored at $4{ }^{0} \mathrm{C}$, a leading cause of TTPI. The parasites survive well in frozen blood (Kark 1982). Plasma that has been frozen or fractionated has never been known to transmit malaria. The incubation period of transfusion malaria depends on the no. and strain of

Pathology Update: Tropical Journal of Pathology \& Microbiology Available online at: www.pathologyreview.in 421 | P a g e 


\section{Original Research Article}

plasmodia transfused, on the host and on the use of antimalarial prophylaxis. The World Health Organization has recognized the urgent need for simple and costeffective diagnostic tests for malaria to overcome the deficiencies of both light microscopy and clinical diagnosis [5]. Traditional practice for outpatients has been to treat presumptively for malaria based on a history of fever but, a significant proportion of those treated may not have parasites (over $50 \%$ in many settings) and hence waste a considerable amount of drugs [6]. This old clinical based practice is still relevant today especially, in infants where time spent on getting a confirmatory laboratory diagnosis could lead to increased fatality.

It has been shown that retinopathy, the study of changes occurring in the retina of the eye, can give good indication of malaria, because the color and other aspects of retina were changed as a result of particular disease.

Various methods for malaria diagnosis are [7]: I. Peripheral smear examination by light microscopy, II. Fluorescence microscopy techniques, III. QBC technique, IV. Non-microscopic Rapid Diagnostic Tests: a) Immunochromatographic tests-detection of malaria antigen by HRP-2 and pLDH detection method, b) Immunochromatographic dipstick assays used for diagnosis - ICT Pf, Para Sight F, and V. Molecular methods: PCR, LAMP technique, Microassay.

The microscopic detection of blood though considered the gold standard for malaria diagnosis, it is quite laborious and requires adequate skill and man power. This had urged the development of other microscopic malarial and rapid diagnostic tests (RDTs) based on the detection of malarial parasite antigen in the blood. RDTs for malaria are based on the detection of either histidine-rich protein 2 (HRP-2), produced only by Plasmodium falciparum, parasite specific lactate dehydrogenase $(\mathrm{pLDH})$ produced by all four species or plasmodium aldolase from the parasite glycolytic pathway, also found in all species.

Up to now, there is no evidence-based guidance to indicate which malaria screening methods are effective for use by transfusion services. More consideration must then be given to post transfusion malaria especially among children under five years old in order to increase the continuous management of childhood illnesses and death.

There are many published studies on prevalence of transfusion transmitted infections, this study particularly focuses on the prevalence of malaria obtained through immunochromatography method and subsequent confirmation of positive cases by gold standard microscopy technique.

\section{Aims and Objectives}

- To evaluate the prevalence of Malaria in eligible blood donors through immunochromatography technique after confirmation by microscopy.

- Study the seasonal variations in the incidence of malaria.

\section{Material and Method}

Place of study- Regional Blood Transfusion Bank Centre, C R Gardi Hospital and R D Gardi Medical College, Ujjain.

Duration of study- February, 2017-April, 2018

Type of study- Cross-sectional and observational study

Inclusion Criteria- The blood donors included in present studywere all replacement and voluntary blood donors. Donors were selected by taking history, clinical examination and following donors' selection criteria according to the Indian FDA rules and regulations for donor selection.

Sample Collection- Written consent was taken. Blood was collected in blood bags containing anticoagulantpreservative solution. Ap proximately $5 \mathrm{ml}$ of donor blood was also collected in two pilot tube (Plain and EDTA) for blood group typing and testing of infectious diseases.

Method- We have routinely screened all donated units of blood for malaria using RDT, based on immunochromatographic methods detecting antigens, histidinerich protein 2 (HRP2- $P$. falciparum), and p-lactate dehydrogenase (pLDH-P. vivax) (MERISCREEN, Mfd. by Meril Diagnostics Pvt. Ltm., one step test for Malaria (Pf/Pv) antigens).

Thick and thin smears were made and examined for parasitic forms for all positive cases, to corroborate the results of RDT.

Additional data analysis was conducted to examine the prevalence trends associated with each infection. Statistical Method- Information regarding donor was extracted from Donor register. Donor register is filled for eligible donors before blood collection. Computerized compilation and coding of collected data was done. All statistical analysis was made by using Stata (version 12, college station, Texas, USA). For 


\section{Original Research Article}

comparing various categorical variables, we used 'Chisquare' test of significance, 'Yates correction' was used at relevant places. 'Z-test' of variation between two means was applied to compare various means at $5 \%$ level of significance. P-value of $<0.05$ was considered statistically significant.

\section{Result}

Total of 10,310 units of donated blood was screened for malaria with two different diagnostic methods between February, 2017 and April, 2018.

Figure A: Prevalence of Malaria antigen among blood donors by immunochromatography (RDT) method. Overall malaria antigen prevalence was estimated at $0.18 \%$ in 10,310 blood donors during February, 2017- April, 2018.

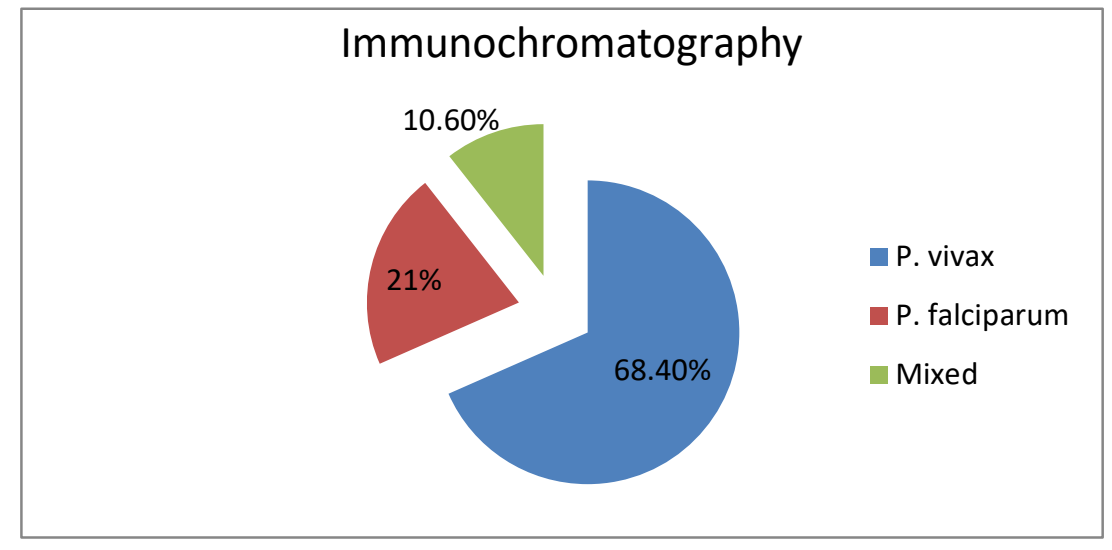

Out of 10,310 blood donors, nineteen blood donors were found to be seropositive for malaria antigen. Of these, 13 blood donors (68.4\%) were positive for P. vivax antigen (Pv-pLDH), 4 blood donors (21\%) were positive for P. falciparum antigen (Pf-HRP2) and 2 blood donors for both antigens (10.6\%). Thus, overall malaria antigen prevalence among blood donors was estimated to be $0.18 \%$. (Fig. A)

Prevalence of malaria among blood donors by microscopy. Overall malaria prevalence was estimated at $0.03 \%$ by microscopy method in 10,310 blood donors during February, 2017- April, 2018. Only 3 out of 10,310 blood donors were found to be positive for malaria through light microscopy in peripheral smear, which makes $0.03 \%$ of total blood donors. Microscopy showed two donors to be positive for P. vivax infection and one for mixed (P. vivax and P. falciparum both) infection while negative for individual $\mathrm{P}$. falciparum infection.

Table-1.1: Comparison of RDT with microscopy method in diagnosis of malariaamong blood donors.

\begin{tabular}{|c|c|c|c|}
\hline RDT & \multicolumn{2}{|c|}{ Microscopy } & Total \\
\hline & Positive & Negative & 19 \\
\hline Positive & 03 & 16 & 10291 \\
\hline Negative & 0 & 10291 & $\mathbf{1 0 3 1 0}$ \\
\hline Total & $\mathbf{0 3}$ & $\mathbf{1 0 3 0 7}$ & $\mathbf{1 0 3}$ \\
\hline
\end{tabular}

Prevalence of malaria antigen with RDT is $0.18 \%$. On confirmation of seropositive blood with light microscopy, only $15.8 \%$ came positive for malaria parasite.

Table-2: Prevalence of malaria among blood donors by microscopy.

\begin{tabular}{|c|c|}
\hline Microscopy & Positive cases(no.) \\
\hline P. vivax & 02 \\
\hline P.falciparum & 00 \\
\hline Mixed & 01 \\
\hline Total & $\mathbf{0 3}$ \\
\hline
\end{tabular}

Overall malaria prevalence was estimated at $0.03 \%$ by microscopy method in 10,310 blood donors during February, 2017- April, 2018 


\section{Original Research Article}

Table-3: Seasonal variation in incidence of malaria among blood donors by two different diagnostic methods.

\begin{tabular}{|c|c|c|}
\hline Season & RDT & Microscopy \\
\hline Winter & 01 & 00 \\
\hline Summer & 03 & 00 \\
\hline Monsoon & 11 & 02 \\
\hline Autumn & 04 & 01 \\
\hline Total & $\mathbf{1 9}$ & $\mathbf{0 3}$ \\
\hline
\end{tabular}

In 10,310 blood donors presented at Blood Bank, R D Gardi Medical College, Ujjain during February, 2017- April, 2018, showing high incidence during rainy season.

On assessment, the three-microscopy confirmed malaria positive blood donors made $15.8 \%$ of total malaria antigen reactive donors. (Table 1.1) None of the donor had given a history of fever/malaria during pre-donation screening.

Seasonal variation was observed in incidence of malaria antigen reactivity. High malaria antigen prevalence was found in rainy season i.e, July-September (57.9\%) followed by summer season i.e, April-June (15.8\%) and autumn i.e, OctoberNovember (21\%), least during winter i.e, December-March (5.3\%).

\section{Discussion}

The prevalence rate of malaria antigen in our donor study population was $0.18 \%$, nineteen donors were tested positive among 10,310 blood donors. Similar to our study, Bahadur et al[4] in their study found malaria antigen prevalence rate of $0.03 \%$ among blood donors by immunochromatographic method. In their study, out of 11,736 units screened, three units were found positive for malarial antigen. Among these three positive samples, two were positive for P.vivax and one was found to be positive for P. falciparum. These three cases were also found to be positive by microscopy.

Hence, they concluded that the use of rapid detection devices along with peripheral smear study of positive donor is a reliable method to prevent transfusion transmitted malaria in India. Anju Dubey et al[8] in their study in northern India reported that none of their donors were found positive by either Microscopy or antigen detection RDT. However, one of the donors who were deferred with history of malaria was found positive by antigen detection RDT and negative by microscopy, which accounts for $0.09 \%$ prevalence rate by antigen detection RDT among blood donors. Therefore, they concluded that blood donor screening by Microscopy may not be an acceptable method.

Malaria antigens currently targeted by RDTs are HRP2, parasite lactate dehydrogenase ( $\mathrm{pLDH})$ and plasmodium aldolase (PL-aldo). Moody et al[9] demonstrated that Plasmodium species secret these proteins thus the sensitivity and specificity of RDTs are measured based on them. P. falciparum has been shown to secret lots of HRP-2 more than HRP-1 and HRP-3 whereas $\mathrm{pLDH}$ and PL-aldo are found in other species of Plasmodium. A number of studies on RDTs have been conducted, although measures of accuracy have varied widely, as a result of differences in methodology, study site epidemiology and type of RDT used i.e. histidine rich protein - 2 (HRP-2) and plasmodium specific lactate dehydrogenase $(\mathrm{pLDH})$ and species specific pLDH or aldolase-based test. [10-12].

A study at New Delhi evaluated the usefulness of new rapid diagnostic test (HRP2/ pLDH Malaria card test) for malaria diagnosis in the forested belt of central India. Their analysis revealed that in comparison to microscopy RDT was 93\% sensitive, $85 \%$ specific with a positive predictive value of $79 \%$ and a Negative predictive value of $95 \%$. (13)

Jessica Martha et al (14) observed false positive Pv$\mathrm{pLDH}$ lines in 6/9 RDTs (including two- three- and four-band RDTs). They occurred in the individual RDT brands at frequencies ranging from $8.2 \%$ to $29.1 \%$. For 19/85 samples, at least two RDT brands generated a false positive Pv-pLDH line. This is of concern as P. falciparum and $\mathrm{P}$. vivax are co-circulating in many regions. The diagnosis of life-threatening $\mathrm{P}$. falciparum malaria may be missed (two-band Pv-pLDH RDT), or the patient may be treated incorrectly with primaquine (three- or four-band RDTs).

Seasonal variations in relation with malaria prevalence can be explained with increased density of vector population during the monsoon and autumn, with increase in breeding fields and favorable conditions for mosquitoes. In present study, nineteen $(0.18 \%)$ donors were found to be malaria antigen positive by RDT, of which only three $(15.8 \%)$ were confirmed by microscopy. None of the donor had given a history of 


\section{Original Research Article}

fever/malaria during pre-donation screening. Seasonal variations were observed. Although RDTs make an important tool for malaria testing, peripheral smear microscopy continues to be the gold standard diagnostic test for malaria diagnosis.

As per experience of many researches and a few published studies, commercially available RDTs lack the consistency, quality control and performance capabilities as claimed by the manufacturers making their use ineffective or potentially dangerous[15].

These data are also in accordance of present study, only three donors tested positive for malaria by microscopic examination of peripheral smear out of nineteen malaria antigen positive donors by RDTs, showing high prevalence of false positive results, concluding that microscopy on peripheral smear is still the most reliable method to diagnose malaria.

\section{Conclusion}

In present study, $19(0.18 \%)$ donors were found to be malaria antigen positive by RDT, of which only three $(15.8 \%)$ were confirmed by microscopy. None of the donor had given a history of fever/malaria during predonation screening. In our study only three donors tested positive for malaria by microscopic examination of peripheral smear as compared to nineteen malaria antigen positive donors by RDTs, showing high prevalence of false positive results, concluding that microscopy on peripheral smear is still the most reliable method to diagnose malaria.

Commercially available RDTs lack the consistency, quality control, high false positive results and performance capabilities as claimed by the manufacturers making their use ineffective or potentially dangerous.

Inference- Although RDT is an important tool for malaria testing in rural settings, we suggest the diagnosis must be confirmed with microscopy method. RDTs can be an important tool for malaria testing, peripheral smear microscopy continues to be the gold standard diagnostic test for malaria diagnosis.

Funding: Nil, Conflict of interest: None initiated

Permission from IRB: Yes

Ethical approval: All procedures performed in studies involving human participants were in accordance with the ethical standards of the institutional and/or national research committee and with the 1964 Helsinki declaration and its later amendments or comparable ethical standards.
Informed consent: Informed consent was obtained from all individual participants included in the study.

\section{References}

1. NACO: Standards for Blood Banks and Blood Transfusion services. New Delhi.: National AIDS Control Organization (NACO), Ministry of Health andFamily Welfare, Government of India. 2007. 108 p.

2. Kitchen AD, Chiodini PL. Malaria and blood transfusion. Vox Sang. 2006 Feb; 90 (2): 77-84. doi:10.1111/j.1423-0410.2006.00733.x

3. Oh JS, Kim JS, Lee CH, et al. Evaluation of a malaria antibody enzyme immunoassay for use in blood screening. Mem Inst Oswaldo Cruz. 2008 Feb;103 (1): 75-8. Epub 2008 Jan 31.

4. Shalini Bahadur, MeenuPujani, and Manjula Jain: Use of rapid detection tests to prevent transfusiontransmitted malaria in India.Asian J Transfus Sci. 2010 Jul; 4(2): 140-141. doi: 10.4103/0973-6247.67033

5. Anonymous: A rapid dipstick antigen capture assay for the diagnosis offalciparum malaria. WHO Informal Consultation on Recent Advances in Diagnostic Techniques and Vaccines for Malaria. Bull World Health Organ 1996, 74:47-54.

6. Shillcutt S, Morel C, Goodman C, et al. Costeffectiveness of malaria diagnostic methods in subSaharan Africa in an era of combination therapy. Bull World Health Organ. 2008 Feb;86(2):101-10.

7. Tangpukdee N, Duangdee C, Wilairatana P, Krudsood S. Malaria diagnosis: a brief review. Korean J Parasitol. 2009 Jun; 47 (2): 93-102. doi: 10.3347/ kjp. 2009.47.2.93. Epub 2009 May 26.

8. Anju Dubey, PritiElhence, Ujjala Ghoshal, and Anupam Verma: Seroprevalence of malaria in blood donors and multi-transfused patients in Northern India: Relevance to prevention of transfusion transmissible malaria. Asian J Transfus Sci. 2012 Jul-Dec; 6(2): 174178. doi: 10.4103/0973-6247.98937

9. Moody A. Rapid diagnostic tests for malaria parasites. Clin Microbiol Rev. 2002 Jan;15(1):66-78.

10. Ashley EA, Touabi M, Ahrer MEvaluation of three parasite lactate dehydrogenase based rapid diagnostic tests for the diagnosis of falciparum and vivax malaria. Malar J. 2009 Oct 27; 8: 241. doi: 10.1186/1475-28758-241. 


\section{Original Research Article}

11. Craig $\mathrm{MH}$, Bredenkamp BL, Williams $\mathrm{CH}$, et al. Field and laboratory comparative evaluation of ten rapid malaria diagnostic tests. Trans R Soc Trop Med Hyg. 2002 May-Jun; 96(3):258-65.

12. Schachterle SE, Mtove G, Levens JP, et al. Prevalence and density-related concordance of three diagnostic tests for malaria in a region of Tanzania with hypoendemic malaria. J Clin Microbiol. 2011 Nov;49 (11): 3885-91. doi: 10.1128/JCM.01157-11. Epub 2011 Aug 31.

13. Bharti PK, Silawat N, Singh PP, et al. The usefulness of a new rapid diagnostic test, the First Response Malaria Combo (pLDH/HRP2) card test, for malaria diagnosis in the forested belt of central India. Malar J. 2008 Jul 11;7:126. doi: 10.1186/1475-28757-126.

14. Maltha J, Gillet P, Cnops L, et al. Malaria rapid diagnostic tests: Plasmodium falciparum infections with high parasite densitiesmay generate false positive Plasmodium vivax pLDH lines. Malar J. 2010 Jul 10;9: 198. doi: 10.1186/1475-2875-9-198.

15. Bell D, Wongsrichanalai C, Barnwell JW. Ensuring quality and access for malaria diagnosis: how can it be achieved? Nat Rev Microbiol. 2006 Sep;4(9 Suppl):S720. doi:10.1038/nrmicro1525

\section{How to cite this article?}

Jain R, Jain P, Kashiv M, P. Desai, U. Chudgar, N. Choudhury, V.K. Mahadik. Comparative evaluation of bivalent malaria rapid diagnostic tests versus traditional microscopy method in assessment of malaria in blood donors at a tertiary care teaching hospital and regional blood transfusion centre in central India. Trop J Path Micro 2018; 4(5):421426.doi:10.17511/jopm.2018.i05.09 